\title{
Allogeneic stem cell transplantation remains an effective therapeutic approach for patients with therapy-related acute myeloid leukemia
}

\author{
Adrianna Spałek, Krzysztof Woźniczka, Anna Armatys, Konrad Matlak, Anna Koclęga, \\ Dariusz Kata, Agata Wieczorkiewicz-Kabut, Grzegorz Helbig*
}

School of Medicine in Katowice, Medical University of Silesia, Department of Hematology and Bone Marrow Transplantation,

Katowice, Poland

\section{Abstract}

Introduction: Therapy-related acute myeloid leukemia (t-AML) remains a late consequence of exposure to cytotoxic chemo- and/or radiotherapy for prior malignant or non-malignant disorders. The prognosis of $t-A M L$ is extremely poor, and allogeneic stem cell transplantation (allo-SCT) seems to be the most effective therapeutic approach. We evaluated the efficacy and safety of allo-SCT for t-AML preceded by solid tumors and lymphomas.

Material and methods: Study patients were retrospectively identified using our institutional database. Nineteen patients (12 female, 7 male), median age 53 years, underwent allo-SCT for t-AML between 2006 and 2018.

Results: Prior malignancy was diagnosed at median age of 43.9 years. Among 19 patients included in the study, 6 (32\%) had prior breast cancer, 2 (11\%) were diagnosed with papillary thyroid cancer, and 2 (11\%) were treated for lymphoma. A variety of other cancers were diagnosed in the remaining 9 patients. Median time from previous malignancy to development of t-AML was 4.9 years. Fourteen patients (74\%) were transplanted in first complete remission (CR1), 4 patients (21\%) were in CR2, and 1 patient received graft being in active disease. 10 patients (53\%) are alive at last contact in $\mathrm{CR}$. Patients died mainly from infectious complications. Median follow-up from prior malignancy and from transplantation was 9.5 years and 1.82 years, respectively. The 2 -year overall survival (OS) was $57 \%$. Median OS for survivors is 4.08 years. Grafts from unrelated donors and the presence of acute graft-versus-host disease affected OS.

Conclusions: Allo-SCT remains an effective therapy for t-AML.

Key words: therapy-related acute myeloid leukemia, solid tumor, lymphoma, allogeneic stem cell transplantation, survival

Acta Haematologica Polonica 2021; 52, 2: 103-109

\section{Introduction}

Therapy-related acute myeloid leukemia (t-AML) is a rare condition that accounts for $1-7 \%$ of all AML cases [1, 2]. It arises as a devastating consequence of prior exposure to radio- or chemotherapy for various solid cancers and/ /or hematologic malignant and non-malignant disorders.
Direct genotoxic damage by prior radiation and chemotherapeutics, as well as the presence of a pre-existing clonal population that remained chemo-resistant, are postulated mechanisms responsible for the development of a new malignancy $[3,4]$. It is estimated that adult patients after oncological treatment have an approximately quadrupled risk of developing AML compared to the general population [1].

\footnotetext{
*Address for correspondence: Grzegorz Helbig, Department of Hematology and Bone Marrow Transplantation, Medical University of Silesia, Dąbrowskiego 25, 40-032 Katowice, Poland, fax: +48 32255 4985, phone: +48 32259 1310, e-mail: ghelbig@o2.pl Received: 20.11.2020 Accepted: 23.11.2020
} 
One may speculate that the incidence of $t-A M L$ is escalating along with the increasing cure rate of solid tumors and lymphomas [5].

According to the 2017 European LeukemiaNet (ELN) recommendations, t-AML should be categorized as a high-risk disease due to its often adverse cytogenetic profile [6]. It is estimated that approximately $50 \%$ of patients with $\mathrm{t}-\mathrm{AML}$ present unfavorable cytogenetic abnormalities including deletions in chromosomes 5, 7 and 17, complex karyotype or translocations involving chromosome 11q23. All these are known to be associated with poor responses to therapy and shorter overall survival [7-9].

Other negative factors predisposing to the development of t-AML include: older age at diagnosis, frequent comorbidities (especially cardiac, renal and liver disfunction), type of prior malignancy, and exposure to specific cytotoxic drugs. Patients with t-AML are usually aged 40-66 [10]. The most common prior malignancy is breast cancer: up to $40 \%$ of cases in some published studies [7, 11, 12]. Among cytostatic agents, prior treatment with alkylating agents and topoisomerase II inhibitors (e.g. etoposide, anthracyclines) is considered to be the most leukemogenic [13]. All these factors may affect the disease outcome and make this patient population of great interest. Despite several novel agents that can be attempted in the treatment of high-risk AML (e.g. midostaurin, venetoclax), allogeneic stem cell transplantation (allo-SCT) still remains the only curative therapeutic approach.

Here, we present our retrospective data on 19 patients with t-AML who underwent allo-SCT.

\section{Material and methods}

Study patients were retrospectively identified through the use of our institutional database of medical records. Therapy-related AML was defined as AML arising at any time after exposure to chemo- and/or radiation therapy for previous solid tumor or lymphoma [9]. Oncological treatment before developing t-AML was considered intense if patients received chemotherapy as well as radiation and non-chemotherapy agents (e.g. monoclonal antibodies, hormonotherapy), moderate if they received chemotherapy and radiation, and mild if they were treated surgically with subsequent radiation [7].

The diagnosis of t-AML, genetic risk stratification and response criteria to therapy were based on the ELN recommendations [6].

Patients were treated according to the Polish Adult Leukemia Group (PALG) protocol with standard induction chemotherapy including $D A \pm C$ regimen (daunorubicin, cytarabine, cladribine). One patient received induction consisting of daunorubicin and etoposide. For those who achieved complete remission (CR), consolidative chemotherapy consisting of high-dose cytarabine was administered. For patients who did not respond to induction or who relapsed after achieving CR1, the following salvage regimens were given: CLAM (cladribine, cytarabine, mitoxantrone), DAC, DA and MEC (mitoxantrone, etoposide, cytarabine).

Response to treatment was assessed after each cycle of chemotherapy as well as before transplantation and at days $+30,+60,+100$, and then every $2-6$ months after the procedure. The response was assessed using cyto-morphological evaluation of bone marrow and measurement of minimal residual disease (MRD) by flow cytometry. Additionally, donor chimerism was assessed by short tandem repeat polymerase chain reaction.

Acute and chronic graft-versus-host disease (GvHD) were graded according to the standard criteria $[14,15]$.

Not all data was available due to the retrospective nature of the study. All patients provided informed consent in accordance with the Declaration of Helsinki.

\section{Statistics}

Time to event was assessed from the day of transplantation. Nonparametric comparisons of group means were performed using the Mann-Whitney $U$ test. Proportions were compared by Fisher exact test. The Kruskal-Wallis test was used to compare more than two independent groups of variables.

Overall survival (OS) was defined as time from day of transplant to death from any cause. The distribution for OS was estimated using Kaplan and Meier method and compared using the log-rank test. A $p<0.1$ was considered significant. Proportional hazard models (Cox regression) were fitted to investigate effects of prognostic factors for OS. All computations were performed with StatSoft Poland analysis software (version 12.0).

\section{Results}

\section{Patient characteristics}

Nineteen patients (12 female, 7 male) with t-AML with a median age of 54 at diagnosis (range 18-70) underwent allo-SCT between 2006 and 2018.

Prior malignancy had been diagnosed at a median age of 43.9 (range 12.9-70.3). Of the 19 patients included in the study, 6 (32\%) had a prior diagnosis of breast cancer, 2 (11\%) were previously diagnosed with papillary thyroid cancer, and 2 (11\%) were treated for lymphoma (1 patient with Hodgkin's disease and 1 with ocular B-cell lymphoma). Different solid cancers were diagnosed in the remaining 9 patients. All the patients were in complete remission (CR) after oncological treatment.

Median time from previous malignancy to development of t-AML was 4.9 years (range $0.93-17.56$ ). Three patients had prior myelodysplastic syndrome. According to the ELN 2017, 6 patients were in the adverse risk category, 10 in the intermediate, and 3 in the favorable [6]. 
Induction regimen consisted of DAC $(n=10), D A(n=8)$ or daunorubicin with etoposide $(n=1)$. Sixteen patients (84\%) achieved first $\mathrm{CR}$, however 3 of them relapsed after consolidation and proceeded to salvage chemotherapy. Three patients remained resistant to induction treatment and required re-induction. In total, 14 patients (74\%) had CR1 at transplant, 4 patients (21\%) were in CR2, and 1 patient was transplanted in active disease. Minimal residual disease was negative in 9 patients (38\%) before procedure and positive in 2 (11\%). In 8 patients (43\%), the results were missing or there was no immunophenotype to follow. Patient characteristics are set out in Table I.

\section{Transplant data}

\section{Baseline characteristics}

\section{of transplanted patients}

Median recipient age was 53 (range 18-70). Median time from diagnosis of t-AML to transplantation was 7.1 months (range 4.9-20.5). Six patients were transplanted from an HLA-matched sibling and 13 patients received either 10/10 HLA-matched unrelated donor ( $n=11$ ) or 9/10 HLA-mismatched grafts $(n=2)$. Peripheral blood was a source of stem cell for 18 patients and one patient received stem cells from bone marrow. In total, myeloablative conditioning (MAC) was used in eight patients, whereas reduced intensity conditioning (RIC) was provided for 11 subjects. MAC consisted of busulfan and cyclophosphamide (BuCy) and fludarabine-based regimens were given as RIC. GvHD prophylaxis included cyclosporine $(n=15)$, cyclosporine with mycophenolate mofetil $(n=3)$, or mycophenolate mofetil alone $(n=1)$.

\section{Outcomes of transplanted patients}

There were no primary graft failures (PGF). Median time to engraftment was 18 days (range 12-26).

Acute and chronic GvHD developed in seven (37\%) and two (11\%) patients, respectively. Acute GvHD grade III/IV occurred in one patient. One patient presented mild and one severe chronic GvHD [15].

Two patients developed life-threatening Enterococci bacteremias (E. cloacae and faecium) in early post-transplant period. One patient developed veno-occlusive disease (VOD). The other complications were mild and included mucositis $(n=7)$, diarrhea $(n=2)$ and fever $(n=2)$.

None of the patients died within 30 days of transplantation, whereas one patient died before day +100 due to septic shock associated with E. cloacae. Post transplantation CR rate was achieved in 18 patients (95\%). One patient relapsed 14 months after transplantation and finally died having failed to respond to salvage chemotherapy.

In total, nine (47\%) patients have died. The main causes of death included pulmonary aspergillosis ( $n=2)$, recurrence of primary malignancy $(n=2)$, ovarian and lung cancer fungal neuroinfection $(n=1)$, AML relapse $(n=1)$
Table I. Patient characteristics

\begin{tabular}{|c|c|}
\hline Variable & $n=19$ \\
\hline Gender (female/male) & $12 / 7$ \\
\hline $\begin{array}{l}\text { Age at diagnosis of prior malignancy, years; } \\
\text { median (range) }\end{array}$ & $\begin{array}{c}43.9 \\
(12.9-70.3)\end{array}$ \\
\hline \multicolumn{2}{|l|}{ Prior malignancy, $\mathrm{n}$ [\%] } \\
\hline Breast cancer & $6(32)$ \\
\hline Papillary thyroid cancer & $2(11)$ \\
\hline Lymphoma & $2(11)$ \\
\hline Colorectal cancer & $1(5)$ \\
\hline Myxoid liposarcoma & $1(5)$ \\
\hline Seminoma & $1(5)$ \\
\hline Urothelial carcinoma & $1(5)$ \\
\hline Pituitary microadenoma & $1(5)$ \\
\hline Cervical cancer & $1(5)$ \\
\hline Ovarian adenocarcinoma & $1(5)$ \\
\hline Endometrial cancer & $1(5)$ \\
\hline Non-small cell lung cancer & $1(5)$ \\
\hline \multicolumn{2}{|l|}{ Treatment of primary malignancy } \\
\hline Intense (hormonotherapy +chemotherapy & $4(21)$ \\
\hline +radiation) & $10(53)$ \\
\hline Moderate (chemotherapy +radiation) & $5(26)$ \\
\hline Mild (radiation) & \\
\hline Hemoglobin level [g/dL]; median (range) & $9.3(6.9-12.8)$ \\
\hline Leukocyte count $\left[\times 10^{9} / \mathrm{L}\right]$; median (range) & $5.2(1.14-45.8)$ \\
\hline 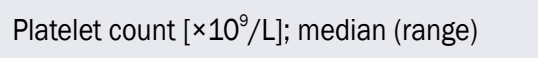 & $49.5(8-182)$ \\
\hline Blasts in blood [\%]; median (range) & $9.5(0-94)$ \\
\hline Blasts in bone marrow (\%); median (range) & $57.5(20-94)$ \\
\hline \multicolumn{2}{|l|}{ Risk group according to ELN, $n$ [\%] } \\
\hline Favorable & $3(15)$ \\
\hline Intermediate & $10(53)$ \\
\hline Adverse & $6(32)$ \\
\hline Prior MDS, n [\%] & $3(15)$ \\
\hline \multicolumn{2}{|l|}{ Hematologic response at transplant, $n$ [\%] } \\
\hline CR1 & $14(74)$ \\
\hline CR2 & $4(21)$ \\
\hline Active disease & $1(5)$ \\
\hline \multicolumn{2}{|l|}{ MRD status before transplant, $\mathrm{n}[\%]$} \\
\hline Positive & $2(11)$ \\
\hline Negative & $9(46)$ \\
\hline Missing & $8(43)$ \\
\hline $\begin{array}{l}\text { Time from t-AML to transplant, months; me- } \\
\text { dian (range) }\end{array}$ & $7.1(4.9-20.5)$ \\
\hline
\end{tabular}

ELN - European LeukemiaNet; MDS - myelodysplastic syndrome; CR1 - first complete remission; CR2 - second complete remission; MRD - minimal residual disease; $\mathrm{t}-\mathrm{AML}$ - therapy-related acute myeloid leukemia 
Table II. Transplant data

\begin{tabular}{|c|c|}
\hline Variable & $n=19$ \\
\hline Age of recipient, median; years (range) & $53(19-71)$ \\
\hline Age of donor, median; years (range) & $34(19-68)$ \\
\hline Donor type, $\mathrm{n}[\%]$ & \\
\hline Related & $6(32)$ \\
\hline 10/10-HLA matched unrelated & $11(58)$ \\
\hline 9/10-HLA mismatched & $2(11)$ \\
\hline Graft source & \\
\hline Peripheral blood & 18 \\
\hline Bone marrow & 1 \\
\hline Myeloablative conditioning, n [\%] & $8(42)$ \\
\hline Conditioning regimen & \\
\hline Busulfan/cyclophosphamide & $8(42)$ \\
\hline Treosulfan/fludarabine & $6(32)$ \\
\hline Busulfan/fludarabine & $5(26)$ \\
\hline $\begin{array}{l}\text { Number of transplanted CD34-positive } \\
\text { cells }\left[\times 10^{6} / \mathrm{kg}\right] \text {; median (range) }\end{array}$ & $5.34(2.7-9.67)$ \\
\hline ANC $>0.5\left[\times 10^{9} / \mathrm{L}\right] ;$ median (range) & $18(12-26)$ \\
\hline 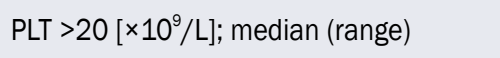 & $13(7-25)$ \\
\hline GvHD prophylaxis, $\mathrm{n}[\%]$ & \\
\hline CsA & $15(79)$ \\
\hline CsA +MMF & $3(16)$ \\
\hline MMF & $1(5)$ \\
\hline Acute GvHD, n [\%] & \\
\hline Grades I-II & $6(32)$ \\
\hline Grades III-IV & $1(5)$ \\
\hline Chronic GvHD, n [\%] & $2(11)$ \\
\hline Hematologic relapse, $n$ [\%] & $1(5)$ \\
\hline Death before day $+100, n[\%]$ & $1(5)$ \\
\hline Alive at last contact, $\mathrm{n}[\%]$ & $10(53)$ \\
\hline $\begin{array}{l}\text { Median follow-up from transplantation, } \\
\text { years; median (range) }\end{array}$ & $\begin{array}{c}1.82 \\
(0.25-13.3)\end{array}$ \\
\hline $\begin{array}{l}\text { Median follow-up from t-AML diagnosis, } \\
\text { years; median (range) }\end{array}$ & $2.36(0.8-13.9)$ \\
\hline $\begin{array}{l}\text { Median follow up from prior malignancy, } \\
\text { years; median (range) }\end{array}$ & $9.5(2.56-20.8)$ \\
\hline
\end{tabular}

HLA - human leukocyte antigens; ANC - absolute neutrophil count; PLT - platelets; GvHD - graft-versus-host disease; CSA - cyclosporin A; MMF - mycophenolate mofetil; t-AML - therapy-related acute myeloid leukemia

and septic shock $(n=1)$. Cause of death remains unknown in two patients.

Ten patients (53\%) are alive at last contact and all remain in CR with full donor chimerism. Median follow-up from diagnosis of prior malignancy, t-AML and transplantation are 9.5 years, 2.36 years, and 1.82 years, respectively.

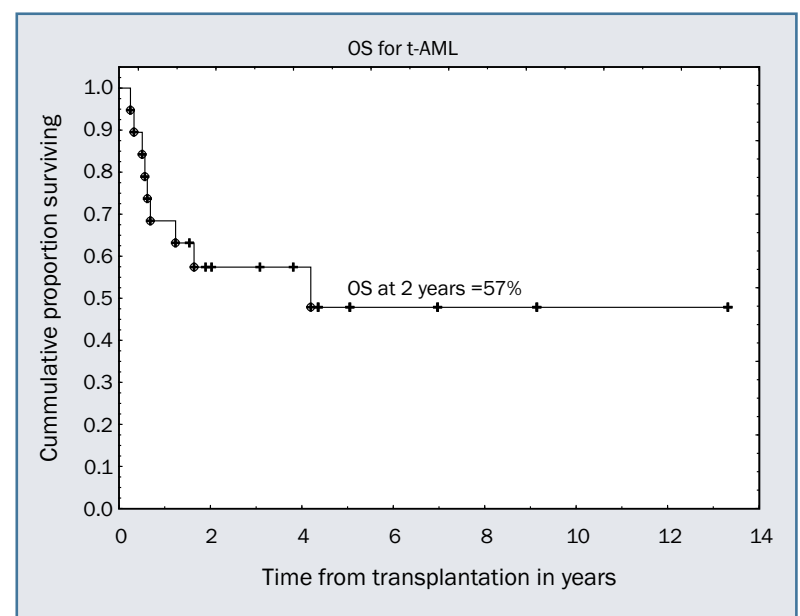

Figure 1. Overall survival (OS) for therapy-related acute myeloid leukemia (t-AML) after transplantation

Table III. Univariate and multivariate analysis of risk factors for overall survival

\begin{tabular}{|c|c|c|c|c|}
\hline \multicolumn{3}{|c|}{ Univariate analysis (log rank) } & \multicolumn{2}{|c|}{$\begin{array}{l}\text { Multivariate analysis } \\
\text { (Cox regression) }\end{array}$} \\
\hline Risk factor & $\begin{array}{c}\text { OS at } 2 \\
\text { years }\end{array}$ & $p$ value & HR $(95 \% \mathrm{Cl})$ & $p$ value \\
\hline $\begin{array}{l}\text { Type of donor } \\
\text { Related }=6 \\
\text { Unrelated }=13\end{array}$ & $33 \%$ & 0.06 & $\begin{array}{c}3.85 \\
(0.97- \\
-15.2)\end{array}$ & 0.05 \\
\hline $\begin{array}{l}\text { Acute GvHD } \\
\text { Yes, } n=7 \\
\text { No, } n=12\end{array}$ & $\begin{array}{l}28 \% \\
57 \%\end{array}$ & 0.09 & $\begin{array}{l}3.73 \\
(0.9- \\
-15.3)\end{array}$ & 0.06 \\
\hline
\end{tabular}

OS - overall survival; HR - hazard ratio; $\mathrm{Cl}$ - confidence interval; GvHD - graft-versus-host disease

Transplant data is summarized in Table II. The 2-year OS was $57 \%$ (Figure 1). Median OS for survivors is 4.08 years (range 1.54-13.3).

Type of donor and presence of acute GvHD had statistically significant impacts on overall survival. Graft from unrelated donor was associated with a better outcome [hazard ratio (HR) 3.85, 95\% confidence interval (Cl): 0.97 $-15.2, p<0.1]$. Presence of acute GvHD (aGvHD) negatively affected OS [HR 3.73, 95\%Cl: 0.9-15.3, $p<0.1]$. Details are shown in Table III.

\section{Discussion}

Therapy related-AML can refer to any leukemic process resulting from previous exposure to leukemogenic chemotherapeutic agents, and this term can be used interchangeably with secondary AML (SAML) $[4,16]$.

The pathophysiology of t-AML is very heterogenous. It has been proved that cytostatics have a direct mutagenic 
impact on DNA, resulting in single- and double-strand breaks in repair processes and chromosomal breakage [17]. Among them, the role of alkylating agents and topoisomerase II inhibitors is well documented. Of note is that these drugs are widely used in the therapy of breast cancer, so it is unsurprising that this neoplasm was the commonest in our cohort, accounting for $32 \%$ of included patients $[11,17]$.

It has also been speculated that hematopoietic malignant clones can be already present at the time of cancer development, and reach their potential to transform into myeloid neoplasms after cytotoxic exposure [4].

It is still unclear whether the well-known poor outcome of t-AML is a result of previous malignancy, or can be explained by other factors such as unfavorable genetic profile or older age. Approximately $50 \%$ of patients with t-AML were found to have adverse karyotype abnormalities in a Danish study, but the long-term outcome remained independent of cytogenetics [2].

Interestingly, patients with core binding factor (CBF) factor t-AML were found to have a worse prognosis than those with de novo AML [18]. The European Bone Marrow Transplantation (EBMT) Group compared the post-transplant outcomes of patients with de novo AML to those transplanted for t-AML. In multivariate analysis, patients with t-AML had lower OS, lower leukemia-free survival (LFS), lower relapse-free survival (RFS), and higher non-relapse mortality (NRM) compared to newly diagnosed AML. There was no difference between the compared groups when de novo AML patients were transplanted in active disease, and the latter remained an independent risk factor for outcome after allo-SCT [19].

A large cohort study reported by the Center for International Bone Marrow Transplant Research (CIBMTR) analyzed 545 patients with t-AML who underwent allo-SCT in order to identify risk factors that negatively affected outcome [20]. The study patients received prior chemotherapy or radiation for solid tumors or hematological malignancies. Median age of the studied population was 40 . Nearly half of the patients had a prior history of lymphoma and $16 \%$ had breast cancer. Only $30 \%$ of individuals had adverse cytogenetics. OS at 5 years was highly unsatisfactory at only $22 \%$ (nota bene: $57 \%$ at 2 years in our study). The following factors had an impact on 0S: age $>35$ years, adverse cytogenetic profile, no remission at transplant, and graft from an unrelated donor. For patients without any of these factors, 5-year OS reached 50\%. In contrast, OS was $4 \%$ in patients presenting all of the abovementioned factors. The incidence of aGvHD was comparable with our study: $39 \%$ versus $37 \%$.

We have proved that OS is negatively affected by the occurrence of aGvHD and grafts from related donors. Disease status at transplantation and type of conditioning did not influence $O S$ in our study. The impact of preparative regimen on the results of transplantation for t-AML with antecedent lymphoma was examined in a study by the EBMT Group. It was demonstrated that patients receiving RIC had a lower risk of NRM and improved LFS compared to those after MAC. OS at 2 years for the entire cohort was 37.4\%. Moreover, patients transplanted in active disease, at older age, with adverse cytogenetics and prior autologous stem cell transplantation (auto-SCT) displayed worse outcomes [21]. If we consider the results of allo-SCT for patients with t-AML preceded by hematological neoplasms and solid tumors, OS and LFS at year 2 were $44.5 \%$ and $38.8 \%$ respectively. Patients receiving MAC regimen had decreased relapse rate, but higher NRM. No differences in terms of OS, LFS and RFS were demonstrated [22].

Another study reported on 65 patients with t-AML/ /MDS following allo-SCT [7]. Median follow-up for survivors was 72 months. OS at 2 years was noticeably lower than in our study (34\% vs. $57 \%$ ), however the study population was slightly different. AML relapse accounted for $41 \%$ of deaths, and this finding was in contrast with our observations, where there was only one fatal relapse among nine deceased patients. On the contrary, our patients died mainly from infectious complications. Unexpectedly, we noticed significantly better OS after transplantation from an unrelated donor. This latter finding is difficult to explain, although the study group was small. The EBMT Group demonstrated lower risk of relapse but higher NRM in patients transplanted from unrelated donors [23].

We demonstrated that the occurrence of acute GVHD negatively influenced survival ( $28 \%$ vs. $57 \%$ at 2 years), and this finding is with line with data presented by the EBMT Group [23].

The leukemogenic role of conditioning regimens used before auto-SCT for lymphoma has also been highlighted by others [24]: it was demonstrated that therapy with alkylating agents and total-body irradiation (TBI) in doses higher than 12 Grey (Gy) increases the risk of t-AML development. Cyclophosphamide-based regimens are proven to be less leukemogenic.

Regarding the lymphoma cases from our study, the outcome was as follows: one patient was primarily diagnosed with ocular B-cell lymphoma at the age of 12 . He was successfully treated with a combination of chemo- and radiotherapy, and t-AML occurred six years later. He is alive 13 years after allo-SCT, being in CR. A second patient developed t-AML 17 years after therapy for Hodgkin's lymphoma. Due to his prior malignancy he received three lines of chemotherapy (ABVD - adriamycin, bleomycin, vinblastine, dacarbazine; BEACOPP - bleomycin, etoposide, doxorubicin, cyclophosphamide, vincristine, procarbazine, and prednisone; and MOPP - mechlorethamine, vincristine, procarbazine, and prednisone) with subsequent radiotherapy. He relapsed within 14 months after allo-SCT and died of resistant leukemia. 
An interesting dilemma is whether a patient with t-AML should be proceeded to auto-SCT. Surprisingly, 3-year OS was comparable between patients who received autoand allo-SCT. Transplant-related mortality was only $12 \%$, although the relapse incidence was $83 \%$ for patients not transplanted in CR. It has been suggested that only young patients transplanted in complete remission may benefit from this procedure [25].

However, it should be remembered that according to the current EBMT recommendations, auto-SCT cannot be considered as a standard of care in this indication, even in children [26]. This can be performed only within clinical trials for individual patients after careful assessment of the potential risks and benefits.

\section{Conclusions}

Despite the low number of included patients and the relatively short follow-up, our study has confirmed an unexpectedly high efficacy of allo-SCT for poor-prognosis patients with t-AML.

\section{Authors' contributions}

AS, GH - planned a study, wrote a manuscript, analyzed data; KW, AA, KM, AK, DK, AWK - collected data, ctitical review.

\section{Conflict of interest None}

\section{Financial support None}

\section{Ethics}

The work described in this article has been carried out in accordance with The Code of Ethics of the World Medical Association (Declaration of Helsinki) for experiments involving humans; EU Directive 2010/63/EU for animal experiments; Uniform Requirements for Manuscripts submitted to Biomedical Journals.

\section{References}

1. Morton LM, Dores GM, Tucker MA, et al. Evolving risk of therapy-related acute myeloid leukemia following cancer chemotherapy among adults in the United States, 1975-2008. Blood. 2013; 121(15): 2996-3004, doi: 10.1182/blood-2012-08-448068, indexed in Pubmed: 23412096.

2. Granfeldt Østgård LS, Medeiros BC, Sengeløv H, et al. Epidemiology and clinical significance of secondary and therapy-related acute myeIoid leukemia: a national population-based cohort study. J Clin Oncol. 2015; 33(31): 3641-3649, doi: 10.1200/JC0.2014.60.0890, indexed in Pubmed: 26304885.

3. Bennett JM. Secondary acute myeloid leukemia. Leuk Res. 1995; 19(4): 231-232, doi: 10.1016/0145-2126(95)00049-t, indexed in Pubmed: 7752670.
4. Chua CC, Fleming S, Wei AH. Clinicopathological aspects of therapy-related acute myeloid leukemia and myelodysplastic syndrome. Best Pract Res Clin Haematol. 2019; 32(1): 3-12, doi: 10.1016/j. beha.2019.02.007, indexed in Pubmed: 30927972.

5. Morton LM, Dores GM, Schonfeld SJ, et al. Association of chemotherapy for solid tumors with development of therapy-related myelodysplastic syndrome or acute myeloid leukemia in the modern era. JAMA Oncol. 2019; 5(3): 318-325, doi: 10.1001/jamaoncol.2018.5625, indexed in Pubmed: 30570657.

6. Döhner H, Estey E, Grimwade D, et al. Diagnosis and management of AML in adults: 2017 ELN recommendations from an international expert panel. Blood. 2017; 129(4): 424-447, doi: 10.1182/ /blood-2016-08-733196, indexed in Pubmed: 27895058.

7. Alam N, Atenafu EG, Kuruvilla J, et al. Outcomes of patients with therapy-related $\mathrm{AML} /$ myelodysplastic syndrome (t-AML/MDS) following hematopoietic cell transplantation. Bone Marrow Transplant. 2015; 50(9): 1180-1186, doi: 10.1038/bmt.2015.151, indexed in Pubmed: 26121109.

8. Wong TN, Ramsingh G, Young AL, et al. Role of TP53 mutations in the origin and evolution of therapy-related acute myeloid leukaemia. Nature. 2015; 518(7540): 552-555, doi: 10.1038/nature13968, indexed in Pubmed: 25487151.

9. Boddu P, Kantarjian HM, Garcia-Manero G, et al. Treated secondary acute myeloid leukemia: a distinct high-risk subset of AML with adverse prognosis. Blood Adv. 2017; 1(17): 1312-1323, doi: 10.1182/ /bloodadvances.2017008227, indexed in Pubmed: 29296774.

10. Madanat YF, Gerds AT. Can allogeneic hematopoietic cell transplant cure therapy-related acute leukemia? Best Pract Res Clin Haematol. 2019; 32(1): 104-113, doi: 10.1016/j.beha.2019.02.011, indexed in Pubmed: 30927969.

11. Kayser S, Döhner K, Krauter J, et al. German-Austrian AMLSG. The impact of therapy-related acute myeloid leukemia (AML) on outcome in 2853 adult patients with newly diagnosed AML. Blood. 2011; 117(7): 2137-2145, doi: 10.1182/blood-2010-08-301713, indexed in Pubmed: 21127174.

12. Shenolikar R, Durden E, Meyer N, et al. Incidence of secondary myelodysplastic syndrome (MDS) and acute myeloid leukemia (AML) in patients with ovarian or breast cancer in a real-world setting in the United States. Gynecol Oncol. 2018; 151(2): 190-195, doi: 10.1016/ /j.ygyno.2018.09.003, indexed in Pubmed: 30268525.

13. Curtis RE, Boice JD, Stovall M, et al. Leukemia risk following radiotherapy for breast cancer. J Clin Oncol. 1989; 7(1): 21-29, doi: 10.1200/ JC0.1989.7.1.21, indexed in Pubmed: 2909667.

14. Glucksberg H, Storb R, Fefer A, et al. Clinical manifestations of graft-versus-host disease in human recipients of marrow from HL-A-matched sibling donors. Transplantation. 1974; 18(4): 295-304, doi: 10.1097/00007890-197410000-00001, indexed in Pubmed: 4153799.

15. Jagasia MH, Greinix H, Arora M, et al. National Institutes of Health Consensus Development Project on Criteria for Clinical Trials in Chronic Graft-versus-Host Disease: I. The 2014 Diagnosis and Staging Working Group Report. Biol Blood Marrow Transplant. 2015; 21(3): 389-401. e1, doi: 10.1016/j.bbmt.2014.12.001, indexed in Pubmed: 25529383.

16. Larson RA. Is secondary leukemia an independent poor prognostic factor in acute myeloid leukemia? Best Pract Res Clin Haematol. 2007; 20(1): 29-37, doi: 10.1016/j.beha.2006.10.006, indexed in Pubmed: 17336252.

17. Cheung E, Perissinotti AJ, Bixby DL, et al. The leukemia strikes back: a review of pathogenesis and treatment of secondary AML. Ann Hematol. 2019; 98(3): 541-559, doi: 10.1007/s00277-019-03606-0, indexed in Pubmed: 30666431. 
18. Borthakur $\mathrm{G}$, Lin $\mathrm{E}$, Jain $\mathrm{N}$, et al. Survival is poorer in patients with secondary core-binding factor acute myelogenous leukemia compared with de novo core-binding factor leukemia. Cancer. 2009; 115(14): 3217-3221, doi: 10.1002/cncr.24367, indexed in Pubmed: 19441109.

19. Schmaelter AK, Labopin M, Socié G, et al. Inferior outcome of allogeneic stem cell transplantation for secondary acute myeloid leukemia in first complete remission as compared to de novo acute myeloid leukemia. Blood Cancer J. 2020; 10(3): 26, doi: 10.1038/s41408020-0296-3, indexed in Pubmed: 32127519.

20. Litzow MR, Tarima S, Pérez WS, et al. Allogeneic transplantation for therapy-related myelodysplastic syndrome and acute myeloid leukemia. Blood. 2010; 115(9): 1850-1857, doi: 10.1182/ /blood-2009-10-249128, indexed in Pubmed: 20032503.

21. Gatwood KS, Labopin M, Savani BN, et al. Transplant outcomes for patients with therapy-related acute myeloid leukemia with prior lymphoid malignancy: an ALWP of EBMT study. Bone Marrow Transplant. 2020; 55(1): 224-232, doi: 10.1038/s41409-019-0673-3, indexed in Pubmed: 31527819.

22. Sengsayadeth S, Labopin M, Boumendil A, et al. Transplant outcomes for secondary acute myeloid leukemia: Acute Leukemia Working Party of the European Society for Blood and Bone Marrow Transplantation Study. Biol Blood Marrow Transplant. 2018; 24(7): 1406-1414, doi: 10.1016/j.bbmt.2018.04.008, indexed in Pubmed: 29678639.
23. Baron F, Labopin M, Savani BN, et al. Graft-versus-host disease and graft-versus-leukaemia effects in secondary acute myeloid leukaemia: a retrospective, multicentre registry analysis from the Acute Leukaemia Working Party of the EBMT. Br J Haematol. 2020; 188(3): 428-437, doi: 10.1111/bjh.16185, indexed in Pubmed: 31612473.

24. Metayer C, Curtis RE, Vose J, et al. Myelodysplastic syndrome and acute myeloid leukemia after autotransplantation for lymphoma: a multicenter case-control study. Blood. 2003; 101(5): 2015-2023, doi: 10.1182/blood-2002-04-1261, indexed in Pubmed: 12393427.

25. Kröger N, Brand R, van Biezen A, et al. Myelodysplastic Syndromes Subcommittee of The Chronic Leukaemia Working Party of the European Group for Blood and Marrow Transplantation (EBMT). Autologous stem cell transplantation for therapy-related acute myeloid leukemia and myelodysplastic syndrome. Bone Marrow Transplant. 2006; 37(2): 183-189, doi: 10.1038/sj.bmt.1705226, indexed in Pubmed: 16299545.

26. Duarte RF, Labopin M, Bader P, et al. European Society for Blood and Marrow Transplantation (EBMT). Indications for haematopoietic stem cell transplantation for haematological diseases, solid tumours and immune disorders: current practice in Europe, 2019. Bone Marrow Transplant. 2019; 54(10): 1525-1552, doi: 10.1038/s41409-0190516-2, indexed in Pubmed: 30953028. 